\title{
Diet composition and its relation to the characteristics of the digestive tract of the blue sea catfish, Ariopsis guatemalensis (Actinopterygii: Ariidae), in Barra de Navidad Lagoon, Jalisco, Mexico
}

\section{Composición de la dieta y su relación con las características del tracto digestivo del chihuil Ariopsis guatemalensis (Actinopterygii: Ariidae) en la laguna Barra de Navidad, Jalisco, México}

\author{
Brenda Daniela Benítez-Mondragón ${ }^{1}$, Consuelo M Aguilar-Betancourt ${ }^{1,2 *}$, Gaspar González-Sansón ${ }^{1,2}$, \\ Gabriela Lucano-Ramírez ${ }^{1}$, Juan Ramón Flores-Ortega ${ }^{3}$, Salvador Ruiz-Ramírez ${ }^{1}$, \\ Daniel Kosonoy-Aceves ${ }^{1}$ \\ ${ }^{1}$ Departamento de Estudios para el Desarrollo Sustentable de Zonas costeras, Universidad de Guadalajara \\ (UDG), Gómez Farías No. 82, CP 48980, San Patricio-Melaque, Jalisco, Mexico. \\ 2 Canadian Rivers Institute, 100 Tucker Park Rd, Saint John, NB E2L 4A6, Canada. \\ 3 Escuela Nacional de Ingeniería Pesquera, Universidad Autónoma de Nayarit, apartado postal no. 10, Bahía de \\ Matanchén km 12, CP 63740, San Blas, Nayarit, Mexico. \\ * Corresponding author. E-mail: consuelo.aguilar@academicos.udg.mx
}

\begin{abstract}
Diet composition and the characteristics of the digestive tract of the blue sea catfish, Ariopsis guatemalensis, in a coastal lagoon in western Mexico were investigated. This species is consumed by locals and it has been used as a bioindicator of sewage pollution. This study aimed to determine if the feeding habits of this species were related to the macroscopic and microscopic morphological characteristics of the digestive tract. Specimens were captured in the Barra de Navidad Lagoon (Jalisco, Mexico) from 2011 to 2016. For every fish collected, total length (TL) was recorded and the esophagus, stomach, and intestine were extracted for histological analyses and identification of food items in their diet. The macroscopic and microscopic characteristics of the digestive tract and the stomach content analysis indicate A. guatemalensis is a carnivorous species with preference for goby fish. Crustaceans were the second most important food item and were dominated by portunid crabs and mud shrimps. The size and position of the mouth, the presence of dental plates on the vomer, palatines, premaxilla, and pharynx, and the presence of goblet cells in the digestive tract are characteristic of fishes that consume live hard-bodied prey and crush their food before it enters the esophagus. The relatively short intestine and the folds in the mucous membrane are characteristic of carnivorous fishes.
\end{abstract}

Key words: feeding habits, Ariopsis guatemalensis, histology, coastal lagoon, digestive tract.

RESUMEN. Se investigaron la composición de la dieta y las características del tracto digestivo del chihuil Ariopsis guatemalensis en una laguna costera en el oeste de México. Esta especie es consumida por habitantes locales, y ha sido utilizada como bioindicadora de contaminación por aguas negras. El objetivo de este estudio fue determinar si los hábitos alimentarios de la especie están relacionados con las características morfológicas macroscópicas y microscópicas del tracto digestivo. Se capturaron los especímenes en la laguna Barra de Navidad (Jalisco, México) de 2011 a 2016. De cada individuo recolectado, se midió la longitud total (LT) y se extrajeron el esófago, el estómago y el intestino para el análisis histológico y la identificación de las entidades alimentarias en su dieta. Según las características macroscópicas y microscópicas del tracto digestivo y el análisis del contenido estomacal, A. guatemalensis tiene hábitos carnívoros y preferencia por los peces góbidos. Los crustáceos fueron la segunda entidad alimentaria más importante, de los cuales destacaron los cangrejos portúnidos y los camarones de fango. El tamaño y la posición de la boca, la presencia de placas dentarias en el vómer, los palatinos, los premaxilares y la faringe, y la presencia de células caliciformes en el tracto digestivo son características de peces que ingieren presas vivas de consistencia dura y fraccionan su alimento antes de que este pase al esófago. El intestino relativamente corto y los pliegues en la mucosa son características de peces carnívoros.

Palabras clave: hábitos alimentarios, Ariopsis guatemalensis, histología, laguna costera, tracto digestivo.

\section{INTRODUCTION}

The Ariidae family has worldwide distribution in tropical and subtropical seas, coastal shelf waters, and the lower basins of coastal rivers and estuaries. Members in this family range from omnivorous to highly specialized feeders (Kobelkowsky and Castillo-Rivera 1995, Mendoza-Carranza 2003, Denadai et al. 2012, Sandoval-Londoño et al. 2015).

\section{INTRODUCCIÓN}

La familia Ariidae se encuentra distribuida mundialmente en mares tropicales y subtropicales, en aguas costeras continentales y en cuencas bajas de ríos costeros y estuarios. $\mathrm{Su}$ alimentación varía desde omnívora hasta muy especializada (Kobelkowsky y Castillo-Rivera 1995, Mendoza-Carranza 2003, Denadai et al. 2012, Sandoval-Londoño et al. 2015). 
Ariopsis guatemalensis belongs to this family and is one of the 92 reported fish species in the Barra de Navidad Lagoon (Jalisco, Mexico) (González-Sansón et al. 2014). This species is of demersal habits (Froese and Pauly 2014) and is capable of bioaccumulating heavy metals (Roosevelt-Rodríguez et al. 2014, Aguilar-Betancourt et al. 2016). No studies have been made on its trophic biology.

Through the evolution of structures and organs involved in the feeding process, species develop the characteristics needed for exploiting resources readily available in the environment (Granado-Lorencio 2002). The overall objective of this project was to study the feeding habits of A. guatemalensis and the relationship between these habits and the main macroscopic and microscopic characteristics of the digestive tract.

\section{MATERIALS AND METHODS}

This study was carried out in the Barra de Navidad Lagoon, which is located on the Pacific coast of western Mexico $\left(19^{\circ} 11^{\prime} 25^{\prime \prime} \mathrm{N}, 104^{\circ} 39^{\prime} 53^{\prime \prime} \mathrm{W}\right)$. The site is a 334-ha-euryhaline-lagoon system that is permanently connected to the sea. The main freshwater supply comes from the Arroyo Seco and the Marabasco Rivers (more details on the study area can be found in Aguilar-Betancourt et al. 2016).

Fish were captured between 2011 and 2016 using different types of artisanal fishing gear: (a) a cast net $3 \mathrm{~m}$ long with a mesh size of $2.5 \mathrm{~cm}$; (b) gill nets $60 \mathrm{~m}$ long with mesh sizes of 7.0, 7.6, 8.9, and $10.2 \mathrm{~cm}$; and (c) an experimental seine net $10 \mathrm{~m}$ long with a mesh size of $1 \mathrm{~cm}$ in the codend. The wide array of fishing techniques was used to reduce any bias introduced by the selectivity of each fishing gear when obtaining the size composition of captured individuals. Each specimen was measured for total length (TL, $\pm 1 \mathrm{~mm}$ precision), and the stomach, esophagus, and intestine were extracted for histological analysis. These organs were measured and fixed in $10 \%$ neutral formaldehyde. Stomach contents were preserved in $70 \%$ ethyl alcohol for subsequent analysis in the laboratory. Prey items were classified, counted, and weighed $( \pm 0.001 \mathrm{~g})$. Sizes were determined by measuring $( \pm 0.1 \mathrm{~cm})$ the total length of shrimp and similar species (from the tip of the rostrum to the rear end of the telson), the total length of fish, and the carapace width of crabs. Organisms were identified to the lowest taxonomic level possible using the literature and specialized keys available for each group.

Prey items were quantified using the numeric method $\left(\% \mathrm{~N}=\mathrm{N}_{\mathrm{i}} / \mathrm{N}_{\mathrm{t}} \times 100\right.$, where $\mathrm{N}_{\mathrm{i}}$ is the number of organisms in category $i$ and $\mathrm{N}_{\mathrm{t}}$ is the total number of organisms in all categories); the gravimetric method $\left(\% \mathrm{~W}=\mathrm{W}_{\mathrm{i}} / \mathrm{W}_{\mathrm{t}} \times 100\right.$, where $\mathrm{W}_{\mathrm{i}}$ is the weight of organisms in category $i$ and $\mathrm{W}_{\mathrm{t}}$ is the total weight of all categories); and the frequency of occurrence $\left(\% \mathrm{FO}=\mathrm{F}_{\mathrm{i}} / \mathrm{N}_{\mathrm{e}} \times 100\right.$, where $\mathrm{F}_{\mathrm{i}}$ is the number of stomachs where category $i$ was found and $\mathrm{N}_{\mathrm{e}}$ is the total number of stomachs analyzed). As a complementary approach, we used the prey-specific index of relative importance (PSIRI,
Ariopsis guatemalensis pertenece a esta familia y es una de las 92 especies de peces registradas dentro de la laguna Barra de Navidad (Jalisco, México) (González-Sansón et al. 2014). Esta especie tiene hábitos demersales (Froese y Pauly 2014) y es capaz de bioacumular metales pesados (Roosevelt-Rodríguez et al. 2014, Aguilar-Betancourt et al. 2016). No se han realizado estudios sobre su biología trófica.

La evolución de las estructuras y órganos involucrados en el proceso de alimentación ha originado que las especies desarrollen las características necesarias para explotar los recursos disponibles en el medio (Granado-Lorencio 2002). El objetivo general de este trabajo fue investigar la alimentación natural de A. guatemalensis y su relación con las principales características macroscópicas y microscópicas del tracto digestivo.

\section{MATERIALES Y MÉTODOS}

La investigación se realizó en la laguna Barra de Navidad, ubicada en la costa mexicana del Pacífico $\left(19^{\circ} 11^{\prime} 25^{\prime \prime} \mathrm{N}\right.$, $\left.104^{\circ} 39^{\prime} 53^{\prime \prime} \mathrm{W}\right)$. El sitio es un sistema lagunar eurihalino de aproximadamente 334 ha que tiene comunicación permanente con el mar. El principal aporte de agua dulce procede de los ríos Arroyo Seco y Marabasco (más detalles sobre el área de estudio se pueden encontrar en Aguilar-Betancourt et al. 2016).

Se realizaron capturas de peces de 2011 a 2016 con distintas artes de pesca artesanales: (a) una atarraya de $3 \mathrm{~m}$ de largo y $2.5 \mathrm{~cm}$ de luz de malla; (b) redes de enmalle de $60 \mathrm{~m}$ de largo y 7.0, 7.6, 8.9 y $10.2 \mathrm{~cm}$ de luz de malla; y (c) un chinchorro playero experimental de $10 \mathrm{~m}$ de largo y $1 \mathrm{~cm}$ de luz de malla en el copo. Con este amplio espectro de métodos de captura, se buscó reducir el sesgo introducido por la selectividad de cada arte de pesca para obtener una composición por tallas de los individuos capturados.

De cada organismo, se midió la longitud total (LT, $\pm 1 \mathrm{~mm}$ de precisión) y se extrajeron el estómago, el esófago y el intestino para el análisis histológico. Estos órganos fueron medidos y fijados en formol neutro al $10 \%$. El contenido estomacal fue preservado en alcohol etílico al $70 \%$ para su posterior análisis en laboratorio. Las entidades alimentarias fueron clasificadas, contadas y pesadas $( \pm 0.001 \mathrm{~g})$. La talla se determinó midiendo $( \pm 0.1 \mathrm{~cm})$ el largo total de los camarones y las especies similares, desde el extremo del rostro hasta el extremo del telson, el largo total de los peces y el ancho del caparazón en los cangrejos. Los organismos fueron identificados hasta el taxón más bajo posible siguiendo la literatura y las guías especializadas para cada grupo.

Las entidades alimentarias fueron cuantificadas de acuerdo con el método numérico $\left(\% \mathrm{~N}=\mathrm{N}_{\mathrm{i}} / \mathrm{N}_{\mathrm{t}} \times 100\right.$, donde $\mathrm{N}_{\mathrm{i}}$ es el número de organismos encontrados de una categoría $i$ y $\mathrm{N}_{\mathrm{t}}$ es el número total de organismos encontrados de todas las categorías); el gravimétrico $\left(\% \mathrm{~W}=\mathrm{W}_{\mathrm{i}} / \mathrm{W}_{\mathrm{t}} \times 100\right.$, donde $\mathrm{W}_{\mathrm{i}}$ es el peso de los organismos de la categoría $i$ y $\mathrm{W}_{\mathrm{t}}$ es el peso total de todas las categorías encontradas); y el de frecuencia 
$\left.\% \mathrm{PSIRI}=\% \mathrm{~F}_{\mathrm{i}} \times \% \mathrm{PN}_{\mathrm{i}}+\% \mathrm{PW}_{\mathrm{i}} / 2\right)$ to determine the importance of each prey (Brown et al. 2012); this index uses $\% \mathrm{~N}$ and $\% \mathrm{~W}$ values fitted to the specific abundance of prey items.

To examine if sample size would provide an estimate that suitably represented stomach contents, cumulative trophic diversity curves were generated following the method proposed by Figueiredo et al. (2005). Trophic diversity was measured using the Shannon index $\left(\mathrm{H}^{\prime}\right)$ (Magurran 2004), which was estimated using 100 randomizations without replacement in EstimateS 9 (Colwell 2017). The curve was considered asymptotic if at least 2 values preceding the trophic diversity value of the total sample $\left(\mathrm{H}_{\text {max }}^{\prime}\right)$ were within the $\mathrm{H}_{\text {max }}^{\prime} \pm 0.05 \mathrm{H}_{\text {max }}^{\prime}$ interval (Alonso et al. 2002).

To analyze if diet composition changed as fish size increased, the total sample was divided into 3 size classes in a way that each class had a similar number of specimens. Size classes were labeled $\mathrm{C} 1$, which included specimens having $\mathrm{TL}<30 \mathrm{~cm}(n=30)$; $\mathrm{C} 2$, which included specimens having TL between 30 and $35 \mathrm{~cm}(n=32)$; and C3, which included specimens having TL $>35 \mathrm{~cm}(n=21)$. Stomach contents were analyzed using the methods mentioned above. Pairwise comparisons of diet composition (\%PSIRI) among size classes were performed using the Bray-Curtis similarity index and the corresponding confidence intervals $(95 \%)$, which were bootstrapped using SpadeR (Chao et al. 2016) in R (R Core Team 2019). Following the criteria by Cumming and Finch (2005) and Gotelli and Colwell (2011), if the confidence intervals for the Bray-Curtis similarity index overlapped between 2 size-class pairs, then differences in diet composition between those classes were not considered significant.

For histological analysis of stomachs, esophagi, and intestines, a tissue sample was taken from each organ, but samples were also taken from the anterior, middle, and posterior intestinal segments. Processing was done following the routine histological technique: samples were dehydrated in alcohol, embedded in paraffin, and sliced with a microtome (Erma, Japan). Tissues were stained with haematoxylin and eosin using the technique by Mayer and Harris, and slices were preserved in Canada balsam (Lucano-Ramírez et al. 2001). Microscopic descriptions were done following GómezRamírez et al. (2010).

\section{Results}

A total of 102 A. guatemalensis stomachs were analyzed, 83 of which had contents. Size data for the analyzed fish were considered homogenous because, although the size interval ranged between 12.3 and $49.0 \mathrm{TL}, 65 \%$ of TL sizes ranged between 25 and $35 \mathrm{~cm}$. The analysis by size classes produced the following Bray-Curtis similarity index values and confidence intervals (in parentheses): $\mathrm{C} 1-\mathrm{C} 2,0.714(0.613-0.814)$; C1-C3, 0.544 (0.442-0.646); C2-C3, 0.636 (0.546-0.727). Confidence intervals overlapped in all cases, so changes in diet composition with specimen size were not considered de ocurrencia $\left(\% \mathrm{FO}=\mathrm{F}_{\mathrm{i}} / \mathrm{N}_{\mathrm{e}} \times 100\right.$, donde $\mathrm{F}_{\mathrm{i}}$ es del número de estómagos donde se encontró la categoría $i$ y $\mathrm{N}_{\mathrm{e}}$ es el número total de estómagos analizados). De manera complementaria, para determinar la importancia de una presa, se empleó el índice de importancia relativa específico para entidades alimentarias (PSIRI, por sus siglas en inglés; \%PSIRI= $\left.\% \mathrm{~F}_{\mathrm{i}} \times \% \mathrm{PN}_{\mathrm{i}}+\% \mathrm{PW}_{\mathrm{i}} / 2\right)$ (Brown et al. 2012), el cual utiliza los valores de $\% \mathrm{~N}$ y $\% \mathrm{~W}$ ajustados a la abundancia específica de las entidades alimentarias.

Para examinar si el tamaño de muestra permitía obtener una estimación representativa del contenido estomacal, se generaron curvas de acumulación de diversidad trófica, según la metodología propuesta por Figueiredo et al. (2005). El índice de Shannon $\left(\mathrm{H}^{\prime}\right)$ (Magurran 2004) se usó para medir la diversidad trófica, y se calculó mediante 100 aleatorizaciones sin remplazo usando el programa EstimateS 9 (Colwell 2017). La curva se consideró asintótica si al menos 2 valores anteriores al de la diversidad trófica de la muestra total $\left(\mathrm{H}_{\max }^{\prime}\right)$ estaban en el intervalo $\mathrm{H}_{\text {max }}^{\prime} \pm 0.05 \mathrm{H}_{\text {max }}^{\prime}$ (Alonso et al. 2002).

Para analizar si hubo un cambio en la composición de la dieta con el incremento de la talla de los peces, la muestra total se dividió en 3 grupos de talla de modo que el número de individuos en cada uno no fuera muy diferente. Estos grupos se denominaron $\mathrm{C} 1$, que incluyó ejemplares con $\mathrm{LT}<30 \mathrm{~cm}$ $(n=30)$; C2, que incluyó ejemplares con LT entre 30 y $35 \mathrm{~cm}$ $(n=32) ; \mathrm{y} \mathrm{C}$, que incluyó ejemplares con LT $>35 \mathrm{~cm}(n=21)$. Se aplicaron los métodos mencionados anteriormente para el análisis del contenido estomacal. La composición de la dieta (\%PSIRI) de los grupos se comparó por pares utilizando el índice de similitud de Bray-Curtis y sus intervalos de confianza (95\%), los cuales se calcularon por técnicas de remuestreo (bootstrap) mediante el programa SpadeR (Chao et al. 2016) en lenguaje $R$ ( $R$ Core Team 2019). Con base en los criterios de Cumming y Finch (2005) y Gotelli y Colwell (2011), se consideró que si los intervalos de confianza del índice de similitud de Bray-Curtis entre 2 pares de grupos de talla se superponían, no había diferencia significativa en la composición de la dieta entre esos grupos.

Para el análisis histológico de los estómagos, esófagos e intestinos, se tomó una muestra de tejido de cada órgano, pero del intestino se tomó una muestra de las partes anterior, media y posterior. El procesamiento se hizo con la técnica histológica de rutina: deshidratación en alcohol, inclusión en parafina, cortes con microtomo (Erma, Japón). Se siguió la técnica de tinción de hematoxilina-eosina de Mayer y Harris, y los cortes se preservaron con bálsamo de Canadá (Lucano-Ramírez et al. 2001). Para la descripción microscópica, se tomó en cuenta lo descrito por Gómez-Ramírez et al. (2010).

\section{RESULTADOS}

Se analizaron un total de 102 estómagos de $A$. guatemalensis, de los cuales 83 presentaron contenido. Los datos de talla de los peces analizados fueron homogéneos, 
significant. Therefore, all subsequent analyses were done considering all fish specimens included in this study.

The number of stomachs analyzed was considered suitable for the description of the species' general diet because the cumulative trophic diversity curve showed a clear tendency to reach the asymptote (Fig. 1). Fishes were the main prey $(\% \mathrm{PSIRI}=53.65, \% \mathrm{~W}=68.51, \% \mathrm{~N}=38.79, \% \mathrm{FO}=$ 74.39). Though most fishes were in advanced stages of digestion, fishes from the Gobiidae family were identifiable, with notable presence of Gobionellus microdon (Gilbert, 1892) $(\%$ PSIRI $=1.84, \% \mathrm{~W}=2.46, \% \mathrm{~N}=1.21, \% \mathrm{FO}=2.44)$ and, to a lesser degree, Ctenogobius manglicola (Jordan \& Starks, 1895) $(\%$ PSIRI $=0.31, \% \mathrm{~W}=0.02, \% \mathrm{~N}=0.61, \% \mathrm{FO}=$ $1.22)$ and Ctenogobius sagittula (Günther, 1862) $(\% \mathrm{PSIRI}=$ $0.31, \% \mathrm{~W}=0.01, \% \mathrm{~N}=0.61, \% \mathrm{FO}=1.22)$. Crustaceans were the second most important prey item $(\% \mathrm{PSIRI}=36.15$, $\% \mathrm{~W}=21.39, \% \mathrm{~N}=50.91, \% \mathrm{FO}=64.63)$. The mud shrimp Upogebia dawsoni (Williams 1986) and the caridean shrimp Alpheus pacificus (Dana 1852) were the most important crustaceans. One peculiar finding was the presence of fish eggs, a prey item with high index values $(\% \mathrm{PSIRI}=7.22$, $\% \mathrm{~W}=7.16, \% \mathrm{~N}=7.27, \% \mathrm{FO}=1.22$; Table 1 ), in one of the A. guatemalensis stomachs.

The sizes of fishes and invertebrates found in stomach contents turned out to be homogeneous in most cases because, although TL intervals were large, standard errors were relatively low and oscillated between $3.6 \%$ and $22.9 \%$ of mean values. When the lengths of prey were compared with the lengths of the fish that contained them in their stomachs, the positive correlation was significant only for specimens of the family Callinectidae (Table 2).

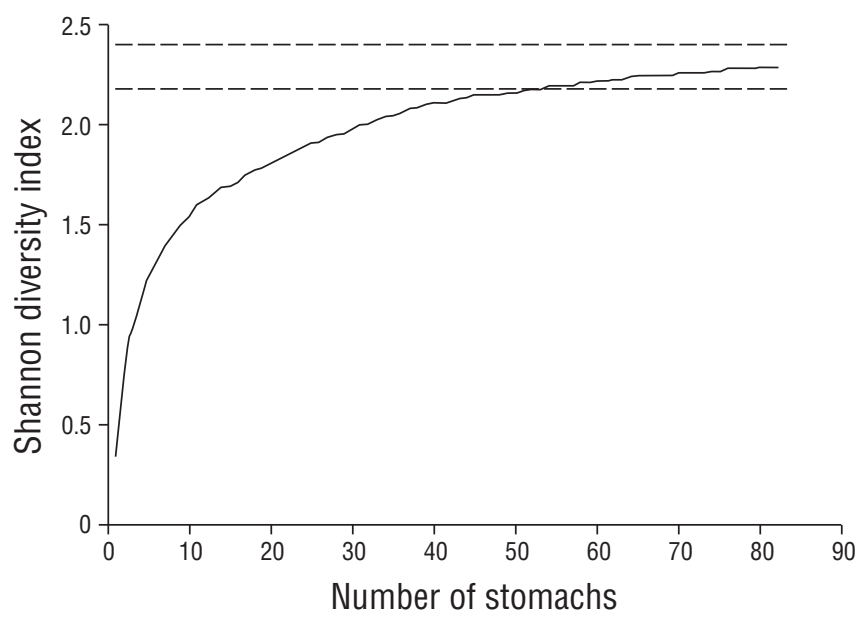

Figure 1. Cumulative trophic diversity curve for Ariopsis guatemalensis. The horizontal dashed lines indicate $\mathrm{H}_{\max }^{\prime} \pm 0.05$ $\mathrm{H}_{\text {max }}^{\prime}$.

Figura 1. Curva de acumulación de diversidad trófica para Ariopsis guatemalensis. Las líneas horizontales discontinuas indican $\mathrm{H}_{\text {max }}^{\prime}$ $\pm 0.05 \mathrm{H}_{\text {max }}^{\prime}$. ya que, aunque el intervalo de tallas fue de 12.3 a $49.0 \mathrm{~cm}$ de LT, el $65 \%$ de las tallas estuvieron entre 25 y $35 \mathrm{~cm}$ de LT. El análisis por grupos de talla dio los siguientes valores del índice de similitud de Bray-Curtis y sus intervalos de confianza (entre paréntesis): $\mathrm{C} 1-\mathrm{C} 2,0.714$ (0.613-0.814); C1-C3, 0.544 (0.442-0.646); C2-C3, 0.636 (0.546-0.727). En todos los casos los intervalos de confianza se superpusieron, por lo que se consideró que no hubo un cambio significativo en la composición de la dieta asociado a la talla de los ejemplares. Con base en este resultado, los análisis posteriores se realizaron para todo el conjunto de peces incluidos en el estudio.

Se consideró que el número de estómagos analizados fue adecuado para describir la dieta general de la especie, pues la curva acumulativa de diversidad trófica mostró una tendencia clara hacia la asíntota (Fig.1). Los peces fueron la presa principal $(\% \mathrm{PSIRI}=53.65, \% \mathrm{~W}=68.51$, $\% \mathrm{~N}=38.79, \% \mathrm{FO}=74.39)$. Aunque la mayoría de los peces se encontraron en un grado de digestión muy avanzado, se reconocieron peces de la familia Gobiidae, de los cuales sobresalieron Gobionellus microdon (Gilbert, 1892) $(\% \mathrm{PSIRI}=1.84, \% \mathrm{~W}=2.46, \% \mathrm{~N}=1.21, \% \mathrm{FO}=2.44) \mathrm{y}$, en menor presencia, Ctenogobius manglicola (Jordan \& Starks, 1895) $(\% \mathrm{PSIRI}=0.31, \% \mathrm{~W}=0.02, \% \mathrm{~N}=0.61, \% \mathrm{FO}=$ 1.22) y Ctenogobius sagittula (Günther, 1862) $(\% \mathrm{PSIRI}=$ $0.31, \% \mathrm{~W}=0.01, \% \mathrm{~N}=0.61, \% \mathrm{FO}=1.22)$. Los crustáceos fueron la segunda entidad alimentaria más importante $(\% \mathrm{PSIRI}=36.15, \% \mathrm{~W}=21.39, \% \mathrm{~N}=50.91, \% \mathrm{FO}=64.63)$. El camarón de fango Upogebia dawsoni (Williams 1986) y el carídeo Alpheus pacificus (Dana 1852) fueron los crustáceos con mayor importancia. Una particularidad encontrada en uno de los estómagos de A. guatemalensis fueron los huevos de pez, entidad que presentó un alto valor en los índices (\%PSIRI $=7.22, \% \mathrm{~W}=7.16, \% \mathrm{~N}=7.27, \% \mathrm{FO}=$ 1.22) (Tabla 1).

Las tallas de los peces y los invertebrados encontrados en el contenido estomacal resultaron homogéneas en la mayoría de los casos, ya que, aunque los intervalos de LT fueron amplios, los errores estándar resultaron relativamente bajos y oscilaron entre el $3.6 \%$ y el $22.9 \%$ de los valores medios. $\mathrm{Al}$ analizar la relación entre el largo de las presas y el largo de los peces que las contenían en sus estómagos, solamente se encontró correlación positiva significativa en el caso de los ejemplares de la familia Callinectidae (Tabla 2).

\section{Descripción macroscópica de Ariopsis guatemalensis}

La boca está en posición subterminal y es relativamente grande. Presenta dientes viliformes, dispuestos en placas: 2 placas premaxilares; 2 placas vomerinas ovaladas, apenas separadas de la línea media y contiguas a 2 placas palatinas; y 2 pares de placas de dientes faríngeos. Presenta 3 pares de barbillas: 1 par maxilar y 2 pares mandibulares. Las branquiespinas son cortas y pocas, con un gran espacio entre ellas. El esófago es corto y corpulento. El estómago es grande y 


\section{Macroscopic description of Ariopsis guatemalensis}

The mouth is in a subterminal position and is relatively large. Teeth are villiform and arranged in plates: 2 premaxillary plates; 2 oval vomerine plates, barely separated from the midline and adjacent to 2 palatine plates; and 2 pairs of pharyngeal tooth plates. It has 3 pairs of barbels: 1 maxillary pair and 2 mandibular pairs. Gill rakers are short and scarce, with large spaces between them. The esophagus is short and bulky. The stomach is large and J-shaped. No pyloric caeca were observed. The intestine is relatively short. The average quotient of total intestine length to total fish length was $1.4 \pm 0.05$ (Fig. 2). en forma de "J". No se observaron ciegos pilóricos. El intestino es relativamente corto. El promedio del cociente del largo total del intestino con respecto al largo total del pez fue de $1.4 \pm 0.05$ (Fig. 2).

\section{Descripción microscópica del tracto digestivo}

Se observaron las 4 capas características del tracto digestivo: mucosa, submucosa, muscular y serosa. En el esófago, la mucosa está compuesta por el epitelio cilíndrico simple y el tejido conectivo laxo; en esta capa se observaron células caliciformes y, en menor cantidad, células alarma. La submucosa está compuesta de tejido conectivo laxo, seguido de una

Table 1. Percentage values of the numerical $(\% \mathrm{~N})$, frequency of occurrence $(\% \mathrm{FO})$, and gravimetric $(\% \mathrm{~W})$ indexes and the prey-specific index of relative importance (\%PSIRI) for the dietary composition of Ariopsis guatemalensis.

Tabla 1. Valores porcentuales de los índices numérico $(\% \mathrm{~N})$, de frecuencia de ocurrencia $(\% \mathrm{FO})$ y gravimétrico $(\% \mathrm{~W})$ y del índice de importancia relativa específico para entidades alimentarias (\%PSIRI) de la composición de la dieta de Ariopsis guatemalensis.

\begin{tabular}{|c|c|c|c|c|c|}
\hline Group & Category & $\% \mathrm{FO}$ & $\% \mathrm{~N}$ & $\% \mathrm{~W}$ & $\%$ PSIRI \\
\hline Porifera & & 4.88 & 1.21 & 2.59 & 1.90 \\
\hline \multirow{2}{*}{ Alpheidae } & Alpheus spp. & 1.22 & 1.21 & 0.31 & 0.76 \\
\hline & Alpheus pacificus & 7.32 & 4.85 & 1.94 & 3.39 \\
\hline Penaeidae & Penaeus californiensis & 2.44 & 1.21 & 0.00 & 0.61 \\
\hline Processidae & Processa spp. & 1.22 & 0.61 & 0.01 & 0.31 \\
\hline \multirow[t]{2}{*}{ Upogebiidae } & Upogebia dawsoni & 7.32 & 16.97 & 2.54 & 9.75 \\
\hline & Shrimp remains & 3.66 & 2.42 & 0.83 & 1.63 \\
\hline Grapsidae & Grapsus spp. & 3.66 & 1.82 & 2.22 & 2.02 \\
\hline \multirow[t]{2}{*}{ Portunidae } & Callinectes spp. & 1.22 & 0.61 & 0.04 & 0.32 \\
\hline & Callinectes arcuatus & 2.44 & 3.64 & 0.45 & 2.04 \\
\hline Xanthidae & Panopeus spp. & 2.44 & 2.42 & 1.13 & 1.78 \\
\hline \multirow[t]{2}{*}{ Leucosiidae } & Randallia bulligera & 1.22 & 0.61 & 0.05 & 0.33 \\
\hline & Brachyuran larvae & 2.44 & 1.21 & 0.02 & 0.61 \\
\hline \multirow[t]{3}{*}{ Paguridae } & & 2.44 & 1.21 & 0.23 & 0.72 \\
\hline & Crab remains & 2.44 & 0.61 & 0.47 & 0.54 \\
\hline & Crustacean remains & 23.17 & 11.52 & 11.16 & 11.34 \\
\hline \multirow[t]{2}{*}{ Insecta } & & 2.44 & 1.82 & 0.35 & 1.08 \\
\hline & Fish eggs & 1.22 & 7.27 & 7.16 & 7.22 \\
\hline \multirow[t]{4}{*}{ Gobiidae } & Ctenogobius sagittula & 1.22 & 0.61 & 0.01 & 0.31 \\
\hline & Ctenogobius manglicola & 1.22 & 0.61 & 0.02 & 0.31 \\
\hline & Gobionellus microdon & 2.44 & 1.21 & 2.46 & 1.84 \\
\hline & Goby remains & 1.22 & 1.21 & 0.44 & 0.83 \\
\hline \multirow[t]{2}{*}{ Engraulidae } & Anchoa spp. & 1.22 & 0.61 & 0.05 & 0.33 \\
\hline & Fish remains & 67.07 & 34.55 & 65.54 & 50.04 \\
\hline
\end{tabular}


Table 2. Mean ( \pm standard error), minimum, and maximum size values for select food categories. The correlation coefficient $(r)$ and its associated probability $(P)$ are given for cases with $N \geq 6$. NI, not identified.

Tabla 2. Valores de talla medios ( \pm error estándar), mínimos y máximos para las categorías alimentarias seleccionadas. Se incluyen los valores del coeficiente de correlación $(r)$ y la probabilidad asociada al mismo $(P)$ para los casos con $N \geq 6$. NI, no identificada.

\begin{tabular}{lrccccc}
\hline Category & $N$ & Mean & Min & Max & $r$ & $P$ \\
\hline Shrimp NI & 3 & $0.83 \pm 0.03$ & 0.80 & 0.90 & - & - \\
Upogebia & 27 & $2.20 \pm 0.12$ & 0.70 & 2.90 & -0.127 & 0.527 \\
Alpheidae & 10 & $2.86 \pm 0.35$ & 1.30 & 4.90 & 0.379 & 0.279 \\
Callinectidae & 7 & $0.36 \pm 0.04$ & 0.20 & 0.50 & 0.816 & 0.025 \\
Crabs NI & 10 & $1.09 \pm 0.21$ & 0.50 & 2.40 & -0.274 & 0.443 \\
Insects NI & 3 & $0.93 \pm 0.13$ & 0.80 & 1.20 & - & - \\
Fishes & 6 & $5.98 \pm 1.37$ & 2.20 & 9.70 & 0.463 & 0.355 \\
\hline
\end{tabular}

\section{Microscopic description of the digestive tract}

The 4 major layers of the digestive tract were observed: mucosa, submucosa, muscular layer, and serosa. In the esophagus, the mucosa is composed of the simple columnar epithelium and loose connective tissue; goblet cells and, in smaller quantities, alarm cells were observed in this layer. The submucosa is composed of loose connective tissue, followed by a circular striated muscle layer (the thicker inner layer) and a longitudinal striated muscle layer (the outer layer) (Fig. 3). In the stomach, the lumen was larger than in other organs (esophagus and intestine). The mucosa is composed of the simple columnar epithelium; gastric glands were observed in this layer. The submucosa is composed of loose connective tissue followed by a circular smooth muscle layer and a longitudinal smooth muscle layer (Fig. 4). Tissue conformation in the intestine was similar. The mucosa is composed of the simple columnar epithelium; unlike in the stomach and the esophagus, no gastric glands or goblet cells were observed here. The submucosa showed lymphoid tissue, followed by a smooth muscle layer with 2 orientations, circular (inner layer) and longitudinal (outer layer) (Fig. 5).

\section{Discussion}

The main prey item consumed by A. guatemalensis was composed of fishes (mainly gobies). Díaz-González and Soto (1988) reported a similar result for Ariopsis caerulescens in the Huizache-Caimanero lagoon system, Sinaloa, Mexico, which predominantly fed on gobies. The results obtained in the present study are similar to those reported for A. caerulescens (Melchor-Aragón 1980) in El Verde Estuary and Caimanero Lagoon in Sinaloa, Mexico, for Bagre marinus (Kobelkowsky and Castillo-Rivera 1995) in lagoons in Veracruz, Gulf of Mexico, and for Ariopsis sp. (aff. assimilis) in a neotropical costal lagoon in Colombia capa de músculo estriado circular (capa interna y de mayor grosor) y una capa de músculo estriado longitudinal (capa externa) (Fig. 3). En el estómago, el lumen fue más grande que en los otros órganos (esófago e intestino). La mucosa está compuesta por el epitelio cilíndrico simple; además, en esta capa se observaron glándulas gástricas. La submucosa está constituida por tejido conectivo laxo, seguido de una capa de músculo liso circular y una capa de músculo liso longitudinal (Fig. 4). La conformación tisular a lo largo del intestino fue similar. La mucosa está compuesta por el epitelio cilíndrico simple; no se observaron glándulas gástricas o células caliciformes, a diferencia del estómago y el esófago. La submucosa presentó tejido linfoide, seguido de una capa de músculo liso en 2 orientaciones, circular (interna) y longitudinal (externa) (Fig. 5).

\section{DiscuSIÓN}

La principal entidad alimentaria de A. guatemalensis estuvo compuesta de peces (principalmente góbidos). Este resultado es similar a lo reportado por Díaz-González y Soto (1988) para Ariopsis caerulescens en el sistema lagunar Huizache-Caimanero, Sinaloa, México, que se alimentó principalmente de peces góbidos. Los resultados obtenidos en este trabajo también son similares a lo reportado para A. caerulescens (Melchor-Aragón 1980) en el estero de El Verde y la laguna de Caimanero en Sinaloa, México, para Bagre marinus (Kobelkowsky y Castillo-Rivera 1995) en lagunas de Veracruz, golfo de México, y para Ariopsis sp. (aff. assimilis) en una laguna costera neotropical en Colombia (Sandoval-Londoño et al. 2015), que consumieron principalmente peces, seguido de crustáceos. Por el contrario, Arius liropus (Melchor 1980) en el estero El Verde y la laguna de Caimanero, Ariopsis canescens en el sistema lagunar Huizache-Caimanero (Díaz-González y Soto 1988), Cathorops melanopus y Ariopsis felis en el golfo de México (Kobelkowsky y Castillo-Rivera 

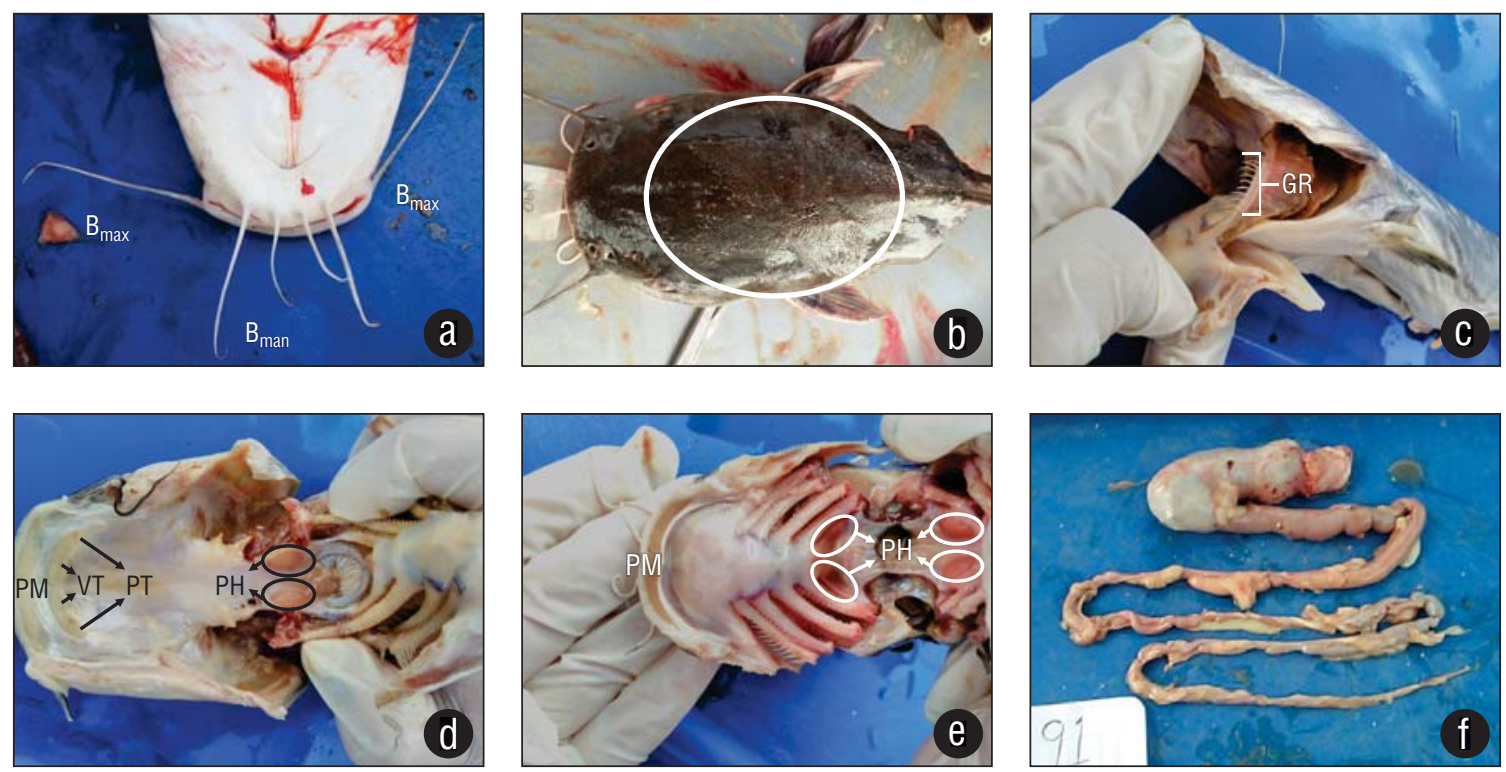

Figure 2. Ariopsis guatemalensis structures. (a) Maxillary barbels $\left(\mathrm{B}_{\mathrm{Max}}\right)$ and mandibular barbels $\left(\mathrm{B}_{\mathrm{Man}}\right)$. (b) Back view of the head (head shield). (c) Gill rakers (GR). (d, e) Vomerine teeth (VT), palatine teeth (PT), premaxillary teeth (PM), and pharyngeal teeth (PH). (f) Digestive tract.

Figura 2. Estructuras de Ariopsis guatemalensis. (a) Barbillas maxilares $\left(\mathrm{B}_{\mathrm{Max}}\right)$ y barbillas mandibulares $\left(\mathrm{B}_{\mathrm{Man}}\right)$. (b) Cabeza en vista dorsal (escudo cefálico). (c) Branquiespinas (GR). (d, e) Dientes vomerinos (VT), dientes palatinos (PT), dientes premaxilares (PM) y dientes faríngeos $(\mathrm{PH})$. (f) Tracto digestivo.
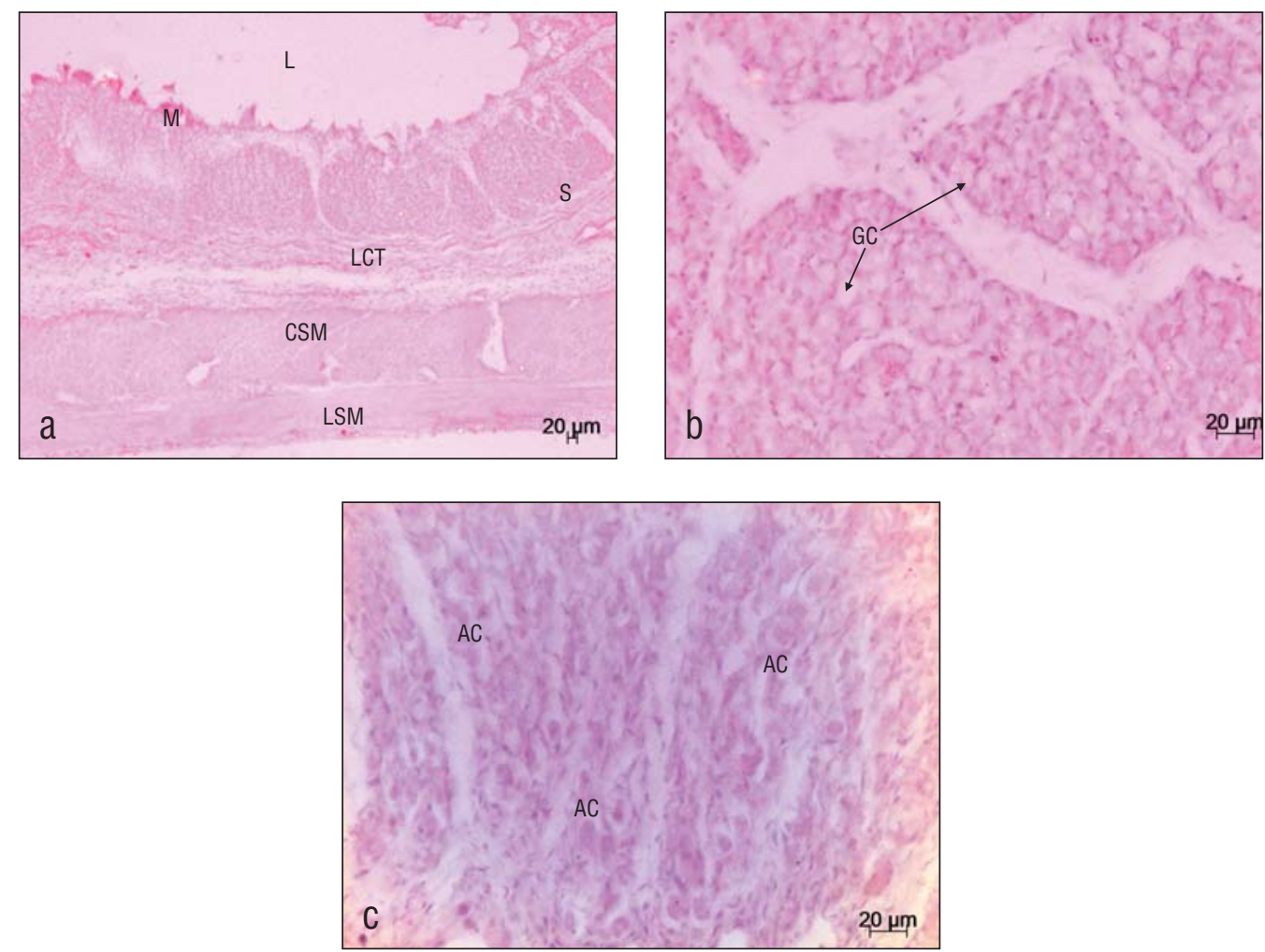

Figure 3. Ariopsis guatemalensis esophagus. (a) Lumen (L), mucosa (M), submucosa (S), loose connective tissue (LCT), circular striated muscle (CSM), and longitudinal striated muscle (LSM) $(5 \times)$. (b) Goblet cells (GC) $(20 \times)$. (c) Alarm cells (AC) $(20 \times)$.

Figura 3. Esófago de Ariopsis guatemalensis. (a) Lumen (L), mucosa (M), submucosa (S), tejido conectivo laxo (LCT), músculo estriado circular $(\mathrm{CSM})$ y músculo estriado longitudinal (LSM) $(5 \times)$. (b) Células caliciformes $(\mathrm{GC})(20 \times)$. (c) Células alarma $(\mathrm{AC})(20 \times)$. 

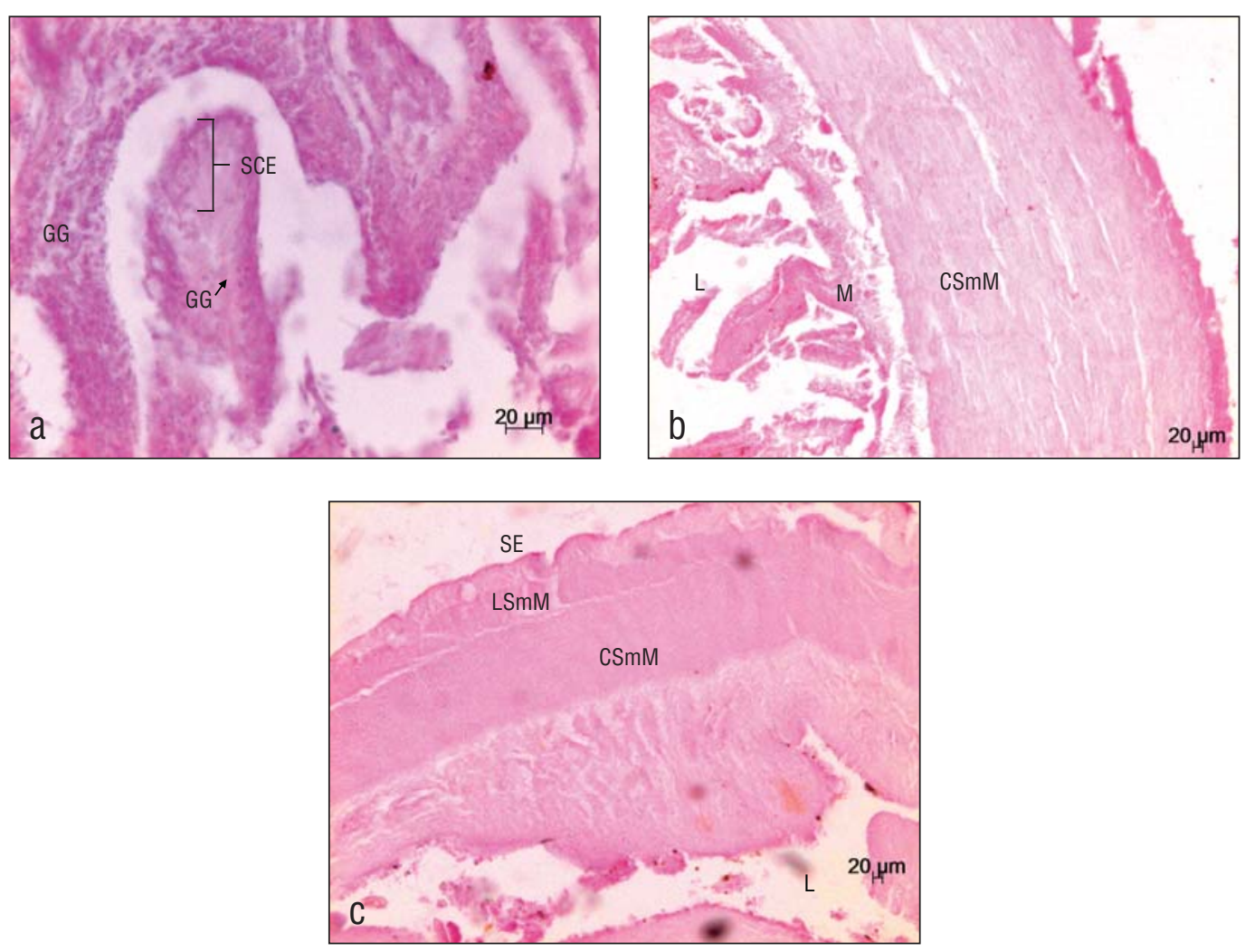

Figure 4. Ariopsis guatemalensis stomach. (a) Simple columnar epithelium (SCE) and gastric glands (GG) (20×). (b) Lumen (L), mucosa $(\mathrm{M})$, and circular smooth muscle $(\mathrm{CSmM})(5 \times)$. (c) Lumen $(\mathrm{L})$, serosa $(\mathrm{SE})$, and longitudinal smooth muscle $(\mathrm{LSmM})(5 \times)$.

Figura 4. Estómago de Ariopsis guatemalensis. (a) Epitelio cilíndrico simple (SCE) y glándulas gástricas (GG) (20×). (b) Lumen (L), mucosa $(\mathrm{M})$ y músculo liso circular $(\mathrm{CSmM})(5 \times)$. (c) Lumen $(\mathrm{L})$, serosa $(\mathrm{SE})$ y músculo liso longitudinal $(\mathrm{LSmM})(5 \times)$.

(Sandoval-Londoño et al. 2015), all which fed mainly on fishes, followed by crustaceans. By contrast, Arius liropus (Melchor 1980) in El Verde Estuary and Caimanero Lagoon, Ariopsis canescens in the Huizache-Caimanero lagoon system (Díaz-González and Soto 1988), Cathorops melanopus and Ariopsis felis in the Gulf of Mexico (Kobelkowsky and Castillo-Rivera 1995), Sciades herzbergii in an estuary north of Brazil (Giarrizzo and Saint-Paul 2008, Krumme et al. 2008), and Ariopsis sp. in the Sinú River in Colombia (Olaya-Nieto et al. 2012) fed mainly on crustaceans, and fishes were their secondary prey.

The absence of change in dietary composition among size classes and the lack of correlation between prey size and fish size can be explained by the fact that this species has a large mouth, which allows individuals, even the smallest ones in the analyzed sample, to capture large-sized prey. The only case for which a significant correlation was found (Callinectidae) should be taken with much caution because the sample size for this case was small $(n=7)$.

The position of the mouth and dental plates enable this species to manipulate live prey and break up food before passing it on to the esophagus. This has been reported by Kobelkowsky and Castillo-Rivera (1995) for C. melanopus,
1995), Sciades herzbergii en un estuario al norte de Brasil (Giarrizzo y Saint-Paul 2008, Krumme et al. 2008) y Ariopsis sp. en el río Sinú en Colombia (Olaya-Nieto et al. 2012) se alimentaron principalmente de crustáceos, y los peces fueron su presa secundaria.

La ausencia de cambios en la composición de la dieta asociados a la talla, y la falta de correlación entre el tamaño de las presas y el tamaño de los peces pueden ser explicadas por el gran tamaño de la boca de esta especie, que le permite capturar presas en un intervalo de tamaños amplio, incluso a los peces más pequeños dentro de la muestra analizada. El único caso en el cual se encontró correlación (Callinectidae) debe ser tomado con mucha cautela debido al pequeño tamaño de la muestra para ese caso $(n=7)$.

La posición de la boca y las placas dentarias permiten el manejo de presas vivas, además de ayudar al fraccionamiento del alimento antes de que este pase al esófago. Esto ha sido reportado por Kobelkowsky y Castillo-Rivera (1995) para C. melanopus, A. felis y B. marinus, las cuales consumen peces y crustáceos, al igual que A. guatemalensis en el presente estudio. Las branquiespinas en A. guatemalensis, al igual que en Ariopsis seemanni (Gómez-Ramírez et al. 2010), no cumplen funciones alimentarias debido al bajo número de 
A. felis, and B. marinus, which feed on fishes and crustaceans, just as A. guatemalensis did in the present study. Gill rakers in A. guatemalensis, as in Ariopsis seemanni (Gómez-Ramírez et al. 2010), do not carry out any digestive function because of the low number of gill rakers and the spacing between them (low filtration capacity).

Ariopsis guatemalensis specimens had relatively short intestines compared to total body length. This concurs with the bentho-icthyophagous habits shown by this species branquiespinas y al espacio entre estas (baja capacidad de filtración).

Los individuos de A. guatemalensis presentaron un intestino relativamente corto en comparación con la longitud total de su cuerpo. Esto concuerda con sus hábitos bento-ictiófagos (Sis et al. 1979, Kramer y Bryant 1995, Wagner et al. 2009, Gómez-Ramírez et al. 2010, Karachle y Stergiou 2010).

El análisis histológico del tracto digestivo de $A$. guatemalensis indicó la presencia de 4 capas: mucosa,
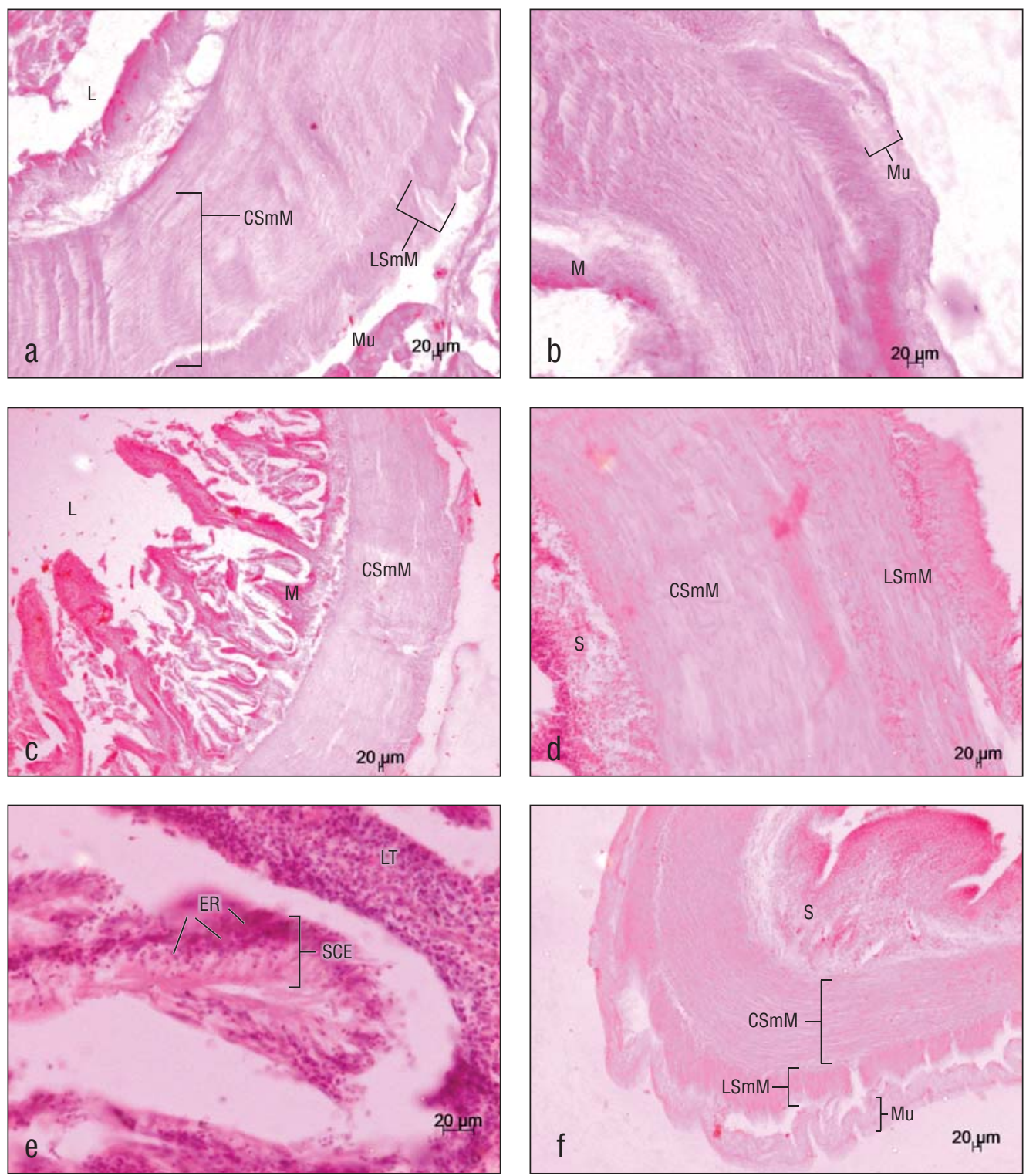

Figure 5. Ariopsis guatemalensis intestine. (a, b) Anterior intestine ( $5 \times$ and $10 \times$, respectively). (c, d, e) Middle intestine $(5 \times, 10 \times$, and $20 \times$, respectively). (f) Posterior intestine $(5 \times)$. Lumen (L), mucosa (M), submucosa (S), longitudinal smooth muscle (LSmM), circular smooth muscle (CSmM), muscular layer (Mu), lymphoid tissue (LT), simple columnar epithelium (SCE), erythrocytes (ER).

Figura 5. Intestino de Ariopsis guatemalensis. (a, b) Intestino anterior ( $5 \times$ y $10 \times$, respectivamente). (c, d, e) Intestino medio $(5 \times$, 10× y $20 \times$, respectivamente). (f) Intestino posterior $(5 \times)$. Lumen $(\mathrm{L})$, mucosa $(\mathrm{M})$, submucosa (S), músculo liso longitudinal (LSmM), músculo liso circular (CSmM), capa muscular (Mu), tejido linfoide (LT), epitelio cilíndrico simple (SCE), eritrocitos (ER). 
(Sis et al. 1979, Kramer and Bryant 1995, Wagner et al. 2009, Gómez-Ramírez et al. 2010, Karachle and Stergiou 2010).

The histological analysis of the digestive tract of A. guatemalensis indicated the presence of 4 layers: mucosa, submucosa, muscularis, and serosa, which coincides with reports for other teleosts, such as Pimelodus pictus (Olaya et al. 2007), A. seemanni (Gómez-Ramírez et al. 2010), Glyptosternum maculatum (Xiong et al. 2011), Rhamdia quelen (Hernández et al. 2009), and Pelteobagrus fulvidraco (Cao and Wang 2009).

The mucosa in the esophagus of A. guatemalensis showed abundant goblet cells, which lubricate and protect the mucosa when food is passed on to the stomach. These cells are a characteristic feature of fish that ingest hardbodied prey (Rodríguez-Rodríguez et al. 2004, Olaya et al. 2007, Gómez-Ramírez et al. 2010). Another feature found in the esophagus of A. guatemalensis was the presence of alarm cells, which according to Olaya et al. (2007), act as immune alarm signals. The layer covering the esophagus showed 2 orientations (circular and longitudinal), indicating that the species has the ability to control regurgitation of unwanted prey or to prevent reflux of gastric contents from the stomach and that there is greater distension of the esophagus during ingestion and movement of food in a single direction (dos Santos et al. 2015).

Enzyme secretion occurs in the stomach, and gastric glands help degrade food before it is passed on to the intestine (Olaya et al. 2007). According to Gómez-Ramírez et al. (2010), the presence of 2 orientations (inner circular and outer longitudinal) in the muscular layers of the stomach allows food to be broken down and mixed with gastric secretions, which results in smaller particles or a semiliquid paste that increases food absorption in the intestine.

The short intestine with mucosal folds in A. guatemalensis is characteristic of fish having carnivorous habits. According to some authors (Sis et al. 1979, Olaya et al. 2007, GómezRamírez et al. 2010), food absorption is proportional to surface area and it increases when there are more mucosal and submucosal folds or apical microvilli. These general considerations adequately describe the characteristics of the intestine in A. guatemalensis.

In conclusion, A. guatemalensis is a benthic carnivorous fish that prefers feeding on fishes and it has adapted to feed on hard-bodied prey (crustaceans). The results obtained here indicate that the characteristics of the digestive tract are strongly related to the feeding habits of A. guatemalensis. The size and position of the mouth, the presence of dental plates on the vomer, the palatine, the premaxilla, and the pharynx, and the presence of goblet cells in the digestive tract are traits in carnivorous fishes that allow them to ingest hard-bodied prey, manipulate live prey, and break up food before it is passed on to the esophagus. The relatively short intestine and mucosal folds in A. guatemalensis are also characteristic of carnivorous fishes. submucosa, muscular y serosa, lo cual coincide con lo reportado para otros teleósteos, como Pimelodus pictus (Olaya et al. 2007), A. seemanni (Gómez-Ramírez et al. 2010), Glyptosternum maculatum (Xiong et al. 2011), Rhamdia quelen (Hernández et al. 2009) y Pelteobagrus fulvidraco (Cao y Wang 2009).

En la mucosa del esófago de A. guatemalensis se encontró una gran cantidad de células caliciformes, las cuales cumplen funciones de lubricación y protección para la mucosa cuando el alimento sea pasado al estómago. Estas células son características de peces que ingieren presas de consistencia dura (Rodríguez-Rodríguez et al. 2004, Olaya et al. 2007, Gómez-Ramírez et al. 2010). Otra característica encontrada en el esófago de A. guatemalensis fue la presencia de células alarma, que de acuerdo con Olaya et al. (2007), actúan como una señal del tipo inmunológica. La capa del esófago presentó 2 orientaciones (circular y longitudinal), lo cual sugiere cierto control para regurgitar presas que no son de interés o para prevenir el reflujo del contenido gástrico del estómago, así como una mayor distención del esófago durante la ingestión y el movimiento del alimento en una sola dirección (dos Santos et al. 2015).

La secreción enzimática ocurre en el estómago, y las glándulas gástricas ayudan a degradar el alimento antes de que este pase al intestino (Olaya et al. 2007). Según Gómez-Ramírez et al. (2010), la presencia de 2 orientaciones (circular interna y longitudinal externa) en las capas musculares del estómago permite una disrupción física del alimento y una mezcla con las secreciones gástricas, lo que da como resultado partículas de menor tamaño o una pasta semilíquida que mejora la absorción de alimentos por parte del intestino.

La presencia en A. guatemalensis de un intestino corto y con pliegues en la mucosa es característico de peces con hábitos carnívoros. Según algunos autores (Sis et al. 1979, Olaya et al. 2007, Gómez-Ramírez et al. 2010), la absorción del alimento es proporcional a la superficie de contacto y aumenta ante la presencia de numerosos pliegues en la mucosa y submucosa o microvellosidades apicales. Estas consideraciones generales explican adecuadamente las características del intestino de A. guatemalensis.

En conclusión, A. guatemalensis es un pez bentónico de hábitos carnívoros con preferencia por los peces, y está adaptada a comer presas de consistencia dura (crustáceos). Los resultados obtenidos indican que existe una gran relación entre las características del tracto digestivo y los hábitos alimentarios de A. guatemalensis. El tamaño y la posición de la boca, la presencia de placas dentarias en el vómer, los palatinos, los premaxilares y la faringe, y la presencia de células caliciformes en el tracto digestivo son características de peces carnívoros que permiten la ingesta de presas de consistencia dura y el manejo de presas vivas, además de ayudar al fraccionamiento del alimento antes de que este pase al esófago. El intestino relativamente corto que presentó A. guatemalensis y los pliegues en la mucosa son también características de peces carnívoros. 


\section{ACKNOWLEDGMENTS}

This study was partially financed by the COECYTJALUDG fund (project 5-2010-1-746) and by the Nuevos Profesores de Tiempo Completo program from the Programa Desarrollo Profesional Docente (103.5/12/3418) (Mexico). We thank all the marine biology students at the University of Guadalajara who volunteered in the fieldwork and laboratory work.

English translation by Claudia Michel-Villalobos.

\section{REFERENCES}

Aguilar-Betancourt CM, González-Sansón G, Kidd KA, Munkittrick KR, Curry RA, Kosonoy-Aceves D, LucanoRamírez G, Ruiz-Ramírez S, Flores-Ortega JR. 2016. Fishes as indicators of untreated sewage contamination in a Mexican coastal lagoon. Mar. Pollut. Bull. 113(1-2): 100-109. https://doi.org/10.1016/j.marpolbul.2016.08.073

Alonso MK, Crespo EA, García NA, Pedraza SN, Mariotti PA, Mora NJ. 2002. Fishery and ontogenetic driven changes in the diet of the spiny dogfish, Squalus acanthias, in Patagonian waters, Argentina. Environ. Biol. Fish. 63(2): 193-202. https://doi.org/10.1023/A:1014229432375

Brown SC, Bizzarro JJ, Cailliet GM, Ebert DA. 2012. Breaking with tradition: redefining measures for diet description with a case study of the Aleutian skate Bathyraja aleutica (Gilbert 1896). Environ. Biol. Fish. 95(1): 3-20. https://doi.org/10.1007/s10641-011-9959-z

Cao XJ, Wang WM. 2009. Histology and mucin histochemistry of the digestive tract of yellow catfish, Pelteobagrus fulvidraco. Anat. Histol. Embryol. 38(4): 254-261. https://doi.org/10.1111/j.1439-0264.2009.00932.x

Chao A, Ma KH, Hsieh TC, Chun-Huo C. 2016. SpadeR: SpeciesRichness Prediction and Diversity Estimation with R. R package version 0.1.1; [accessed 2018 Jul 03]. https://CRAN.R-project.org/package=SpadeR

Colwell R K. 2017. EstimateS: Statistical Estimation of Species Richness and Shared Species from Samples. Version 9. User's Guide and Application published. [Unknown]: [publisher unknown]; [accessed 2018 Jul 01]. http://viceroy.eeb.uconn.edu/ estimates/

Cumming G, Finch S. 2005. Inference by eye: confidence intervals and how to read pictures of data. Am. Psychol. 60(2): 170-180. http://dx.doi.org/10.1037/0003-066X.60.2.170

Denadai MR, Bessa E, Borges-Santos F, Silva-Fernandez W, Motta-da-Costa-Santos F, Malagutti-Feijó M, Dias-Arcuri AC, Turra A. 2012. Life history of three catfish species (Siluriformes: Ariidae) from southeastern Brazil. Biota Neotrop. 12(4): 74-83. http://dx.doi.org/10.1590/S1676-06032012000400008

Díaz-González G, Soto LA. 1988. Hábitos alimenticios de peces depredadores del sistema Lagunar Huizache-Caimanero, Sinaloa, México. An. Inst. Cienc. Mar Limnol. 15: 97-124.

Dos Santos ML, Arantes FP, Pessali TC, dos Santos JE. 2015. Morphological, histological and histochemical analysis of the digestive tract of Trachelyopterus striatulus (Siluriformes: Auchenipteridae). Zoologia (Curitiba) 32(4): 296-305. http://dx.doi.org/10.1590/S1984-46702015000400005

Figueiredo M, Morato T, Barreiros JP, Afonso P, Santos RS. 2005. Feeding ecology of the white seabream, Diplodus sargus, and

\section{Agradecimitentos}

Esta investigación fue financiada parcialmente por el fondo COECYTJAL-UDG (proyecto 5-2010-1-746) y por el programa Nuevos Profesores de Tiempo Completo del Programa para el Desarrollo Profesional Docente (103.5/12/3418) (México). Agradecemos a los estudiantes de Biología Marina de la Universidad de Guadalajara que participaron como voluntarios en el trabajo de campo y de laboratorio.

the ballan wrasse, Labrus bergylta, in the Azores. Fish. Res. 75(1-3): 107-119. https://doi.org/10.1016/j.fishres.2005.04.013

Froese R, Pauly D, editors. 2014. FishBase: World Wide Web electronic publication; [accessed 2018 Oct 02]. http://www. fishbase.org.

Giarriozzo T, Saint-Paul U. 2008. Ontogenetic and seasonal shifts in the diet of the pemecou sea catfish Sciades herzbergii (Siluriformes: Ariidae), from a macrotidal mangrove creek in the Curuçá estuary, Northern Brazil. Rev. Biol. Trop. 56(2): 861-873. https://doi.org/10.15517/rbt.v56i2.5629

Gómez-Ramírez E, Tovar-Bohórquez MO, Obando-Bulla MJ, Hurtado-Giraldo H. 2010. Estudio histológico del tracto digestivo del pez Ariopsis seemanni (Ariidae). Rev. Fac. Cienc. Básicas 6(2): 216-225.

González-Sansón G, Aguilar-Betancourt C, Kosonoy-Aceves D, Lucano-Ramírez G, Ruiz-Ramírez S, Flores-Ortega JR, Hinojosa-Larios Á, Silva-Bátiz FA. 2014. Composición por especies y tallas de los peces en la laguna Barra de Navidad, Pacífico central mexicano $=$ Species and size composition of fishes in Barra de Navidad lagoon, Mexican central Pacific. Rev. Biol. Trop. 62(1): 129-144. https://doi.org/10.15517/rbt.v62i1.10001

Gotelli NJ, Colwell RK. 2011. Estimating species richness. In: Magurran AE, McGill BJ (eds.), Biological Diversity: frontiers in measurement and assessment. United Kingdom: Oxford University Press; p. 39-54.

Granado-Lorencio C. 2002. Ecología de Peces. Sevilla (Spain): Editorial Universidad de Sevilla; 356 pp.

Hernández DR, Pérez-Gianeselli M, Domitrovic HA. 2009. Morphology, histology and histochemistry of the digestive system of South American catfish (Rhamdia quelen). Int. J. Morphol. 27(1): 105-111. https://doi.org/10.4067/s0717-95022009000100019

Karachle PK, Stergiou KI. 2010. Gut length for several marine fish: relationships with body length and trophic implications. Mar. Biodivers. Rec. 3: 1-10. https://doi.org/10.1017/S1755267210000904

Kobelkowsky AD, Castillo-Rivera M. 1995. Sistema digestivo y alimentación de los bagres (Pisces: Ariidae) del Golfo de México. Hidrobiológica 5(1-2): 95-103.

Kramer DL, Bryant MJ. 1995. Intestine length in the fishes of a tropical stream: 2. Relationships to diet-the long and short of a convoluted issue. Environ. Biol. Fish. 42(2): 129-141. https://doi.org/10.1007/BF00001991

Krumme U, Brenner M, Saint-Paul U. 2008. Spring-neap cycle as a major driver of temporal variations in feeding of intertidal fishes: Evidence from the sea catfish Sciades herzbergii 
(Ariidae) of equatorial west Atlantic mangrove creeks. J. Exp. Mar. Biol. Ecol. 367(2): 91-99. https://doi.org/10.1016/j.jembe.2008.08.020

Lucano-Ramírez G, Villagrán-Santa Cruz M, Ruiz-Ramírez S, López-Murillo T. 2001. Histology of the oocytes of Lutjanus peru (Nichols and Murphy, 1922) (Pisces: Lutjanidae) = Histología de los ovocitos de Lutjanus peru (Nichols y Murphy, 1992). (Pisces: Lutjanidae). Cienc. Mar. 27(3): 335-349. http://dx.doi.org/10.7773/cm.v27i3.493

Magurran AE. 2004. Ecological diversity and its Measurements. Oxford: Blackwell Publishing; 256 pp.

Melchor-Aragón JM. 1980. Estudio sobre la biología y ecología de los chihuiles Arius caerulescens Günther y Arius liropus (Bristol) del estero de El Verde y Laguna de Caimanero, Sinaloa (Pisces: Ariidae) [BSc thesis]. [La Paz (Baja California Sur, Mexico)]: Instituto Politécnico Nacional, Centro Interdisciplinario de Ciencias Marinas. http://repositoriodigital. ipn.mx/handle/123456789/16101

Mendoza-Carranza M. 2003. The feeding habits of gafftopsail catfish Bagre marinus (Ariidae) in Paraiso Coast, Tabasco, Mexico. Hidrobiológica 13(2): 119-126.

Olaya CM, Ovalle CH, Gómez E, Rodríguez D, Caldas ML, Hurtado H. 2007. Histología y morfometría del sistema digestivo del silúrido bagre tigrito (Pimelodus pictus) = Histology and morphology of the digestive system of bagre tigrito (Pimelodus pictus). Rev. Fac. Med. Vet. Zoot. 54: 311-323.

Olaya-Nieto CW, Arellano-Padilla JJ, Martínez-González AL. 2012. Hábitos alimentarios del barbul de piedra (Ariopsis sp.) en el río Sinú, Colombia $=$ Feeding habits of Barbul de Piedra (Ariopsis sp.) in the Sinu River, Colombia. Acta Biol. Colomb. 17(1): 117-128.

R Core Team. 2019. R: A language and environment for statistical computing. Vienna (Austria): R Foundation for Statistical Computing. https://www.R-project.org/.

Rodríguez-Rodríguez J, González E, Hernández-Contreras N, Capó V, García I. 2004. Comparación morfológica e histológica del tubo digestivo de Gambusia puncticulata y Girardinus metallicus, (Cyprinodontiformes: Poeciliidae), peces utilizados en el control biológico de mosquitos. Rev. Cubana Med. Trop. 56(1): 73-76.

Roosevelt-Rodríguez A, Monks S, Pulido-Flores G, GaytánOyarzún JC, Romo-Gómez C. 2014. Presencia de Plomo y Cadmio en Ariopsis guatemalensis (Günter, 1864), en la laguna de Tres Palos, Guerrero, México $=$ Presence of Lead and Cadmium in Ariopsis guatemalensis (Günter, 1864), in the Tres Palos Lagoon, Guerrero, Mexico. Revista Biológico Agropecuaria 2(3): 551-555.

Sandoval-Londoño L, Leal-Flórez J, Blanco-Libreros JF, Taborda-Marín A. 2015. Hábitos alimenticios y aspectos del uso del hábitat por el chivo cabezón Ariopsis sp. (aff. assimilis) (Siluriformes: Ariidae), en una laguna costera neotropical (Ecorregión Darién, Colombia) $=$ Food habits and aspects of habitat use by the catfish Ariopsis sp. (aff. assimilis) (Siluriformes: Ariidae) in a Neotropical coastal lagoon (Darien Ecoregion, Colombia). Actual. Biol. 37(102): $55-66$.

Sis RF, Ives PJ, Jones DM, Lewis DH, Haensly WE. 1979. The microscopic anatomy of the oesophagus, stomach and intestine of the channel catfish, lctalurus punctatus. J. Fish Biol. 14(2): 179-186. https://doi.org/10.1111/j.1095-8649.1979.tb03508.x

Wagner CE, McIntyre PB, Buels KS, Gilbert DM, Michel E. 2009. Diet predicts intestine length in Lake Tanganyika's cichlid fishes. Funct. Ecol. 23(6): 1122-1131. https://doi.org/10.1111/j.1365-2435.2009.01589.x

Xiong D, Zhang L, Yu H, Xie C, Kong Y, Zeng Y, Huo B, Liu Z. 2011. A study of morphology and histology of the alimentary tract of Glyptosternum maculatum (Sisoridae, Siluriformes). Acta Zool. 92(2): 161-169. https://doi.org/10.1111/j.1463-6395.2010.00458.x

Received April 2019, accepted October 2019. 